\title{
Students' Reasoning Behavior on Generalization of Figural Pattern
}

\author{
Rizki Ayu Triwardani, Yusuf Fuad and Masriyah \\ Program Pascasarjana, Department of Mathematics, Universitas Negeri Surabaya, Ketintang, Surabaya 60231, East \\ Java, Indonesia \\ rizkitriwardani16070785004@mhs.unesa.ac.id
}

\begin{abstract}
Pattern generalization is a core area in mathematics that is recognized by a community of researchers as an approach to develop student's algebraic reasoning. Examining students' generalization strategies of patterns becomes very important in term of learning advanced algebra. This study applied qualitative descriptive methods and was to investigate of students' reasoning behavior to generalize some algebraic patterns. The sample were the $8^{\text {th }}$ grade students of SMPN 2 Jatirejo Mojokerto. Two volunteer students (first and second) were selected as research subjetcs and based on the existing of students reasoning behaviors. The semi-structured interview were individually conducted to reveal students reasoning behaviors in constructing generalize patterns while they solved the given problem. The obtained data was classified through the generalization strategies in the existing literatures. According to the results of the task performed by the first subject, she performed figural reasoning based on the figural pattern task. The second subject categorized numerical reasoning based on the figural pattern task. The result of this study emphasized that students mainly utilized a recursive strategy to achieve immediate and near generalization. Two of subjects used functional strategy to reach the far generalization but had not yet received the correct answer. The expected implications of this study are as consideration for designing the learning on the generalization of patterns in accordance with the students' reasoning behavior.
\end{abstract}

Keywords- reasoning behavior, generalization, figural pattern, immediate generalization, near generalization, far generalization

\section{INTRODUCTION}

Reasoning is a fundamental aspect of mathematics [1]. Reasoning is a very important aspect of mathematical ability in teaching and learning of mathematics. Reasoning acts basic role and capability to improve general mathematical abilities [2]. In mathematics, reasoning is utilized to solve problems and also to decide whether an assertion (e.g. an answer to a problem) is correct [3]. The power of mathematics lies in relation and transformation which give rise to pattern and generalization [4].

Generalization implies such deliberate reasoning that builds on specific cases to identify inter-model, inter- procedural or inter-structural relationships. We can link generalizing to mathematical reasoning because the process is clearly associated with inference and discourse, without necessarily creating a new incommensurable discourse [4]. Generalization plays a crucial role in the activity of any mathematician, being considered an inherent ability to mathematical thinking in general [5].

Patterns is a fundamental stage in the formation of generalization. Pattern generalization is a core area in mathematics that is recognized by a community of researchers as an approach to develop student's algebraic reasoning [6]. Patterning is critical to the abstraction of mathematical ideas and relationships, and the development of mathematical reasoning in young children. Examining students' generalization strategies of patterns becomes very important in term of learning advanced algebra.

The importance given to visualization in the learning of mathematics is based on the fact that it's not confined to mere illustration of ideas, but it's also recognized as a component of reasoning. Generalizations based on the study of figural patterns allow students to contact with the dynamic component of the conceptual construction of mathematical objects and concepts (Rivera, 2007) and more easily assign meaning to symbols and expressions.

In the context of visual patterns, students that are able to analyse figures discursively, can do it in different ways: identify disjoint sets of elements that are combined to build the initial figure, using a constructive generalization; observe the existence of overlapping subsets, counting certain elements more than once that are subsequently subtracted, which means that the generalization is formulated in a deconstructive way [7]. Several studies have concluded that students tend to use more frequently constructive generalizations than deconstructive ones $[7,8]$ since the latter category involves a higher cognitive level regarding visualization. 


\section{METHOD}

This study applied qualitative descriptive methods and was to investigate of students' reasoning behavior to generalize some algebraic patterns. An instrument was designed for this study and was validated by two mathematics lecturers. One problem related to figural patterns were included as a tool of data collection. The problem consisted of six questions. The questions were based on the type of generalization. The type of generalization was immediate generalization, near generalization, and far generalization. Immediate generalization was addressed in point $a, b$ in each problems. Near generalization was referred to point $c, d$ in each problems. Far generalization was related to point $e, f$ in each problems.

The sample were the $8^{\text {th }}$ grade students of SMPN 2 Jatirejo Mojokerto. All students were given the algebraic problems related to figural patterns from which the results were categorized into three mathematics levels, i.e. high, medium, and low mathematics level respectively. Two volunteer students (first and second) were selected as research subjetcs and based on the existing of students reasoning behaviors. The semi-structured interview were individually conducted to reveal students reasoning behaviors in constructing generalize patterns while they solved the given problem. The obtained data was classified through the generalization strategies in the existing literatures. Table 1 shows the indicators of students reasoning behavior on generalization of figural pattern.

TABLE I. INDICATOR OF STUDENTS REASONING BEHAVIOR ON GENERALIZATION OF FIGURAL PATTERN

\begin{tabular}{|c|c|c|}
\hline \multicolumn{1}{|c|}{$\begin{array}{c}\text { Strategy } \\
\text { Constructed } \\
\text { figure }\end{array}$} & $\begin{array}{c}\text { Numerical } \\
\text { Reasoning } \\
\text { in an incorrect way } \\
\text { that does not follow } \\
\text { the growth of the } \\
\text { pattern and then } \\
\text { counts its elements. }\end{array}$ & $\begin{array}{c}\text { Figural Reasoning } \\
\text { fonstructs a figure in a } \\
\text { following the growth of the } \\
\text { pattern and then counts its } \\
\text { elements. }\end{array}$ \\
\hline Recursive & $\begin{array}{l}\text { Recognizes a } \\
\text { numeric pattern by } \\
\text { finding the } \\
\text { difference between } \\
\text { consecutive terms } \\
\text { and then adds it to a } \\
\text { numeric value in } \\
\text { given step to reach } \\
\text { next step. }\end{array}$ & $\begin{array}{l}\text { Recognizes the structural } \\
\text { growth of the pattern } \\
\text { between consecutive figural } \\
\text { steps and then adds the value } \\
\text { of the growth to a given term } \\
\text { in order to reach the next } \\
\text { figural step. }\end{array}$ \\
\hline Whole-object & $\begin{array}{l}\text { Multiplies the value } \\
\text { of a step to find the } \\
\text { numeric value of } \\
\text { another step. }\end{array}$ & $\begin{array}{l}\text { Multiplies parts of the pattern } \\
\text { in step a by a number } \mathrm{n} \text { such } \\
\text { that } \mathrm{n} \times \text { a is the required } \\
\text { figural step number. }\end{array}$ \\
\hline
\end{tabular}

\begin{tabular}{|c|c|c|}
\hline Strategy & $\begin{array}{l}\text { Numerical } \\
\text { Reasoning }\end{array}$ & Figural Reasoning \\
\hline Chunking & $\begin{array}{l}\text { Identifies a constant } \\
\text { difference } \\
\text { between pairs of } \\
\text { consecutive steps } \\
\text { of the pattern. Uses } \\
\text { this difference } \\
\text { to multiply it by the } \\
\text { number of } \\
\text { steps needed to } \\
\text { reach the required } \\
\text { step in the pattern. }\end{array}$ & $\begin{array}{l}\text { Distinguishes the structural } \\
\text { growth of the pattern } \\
\text { between consecutive figural } \\
\text { steps, counts the number of } \\
\text { steps needed to reach the } \\
\text { required figural step in the } \\
\text { pattern, multiplies the } \\
\text { number of steps needed with } \\
\text { the constant difference } \\
\text { between consecutive figural } \\
\text { steps and then adds the result } \\
\text { to the given figural step. }\end{array}$ \\
\hline Functional & $\begin{array}{l}\text { Recognizes a rule } \\
\text { based on the } \\
\text { numeric pattern. }\end{array}$ & $\begin{array}{l}\text { Identifies the growing } \\
\text { components of the pattern as } \\
\text { well as the constant } \\
\text { components and relates them } \\
\text { with each other and with the } \\
\text { figural step number. }\end{array}$ \\
\hline
\end{tabular}

\section{RESULTS}

The algebraic problems related to figural patterns task was analyzed based on indicators. Based on the analysis results there were 3 students with the high mathematical level, medium mathematical level, and low mathematical level.

A. The reasoning of first subject (high mathematical level)

Problem at generalization task was solved by first subject. The first subject answered the immediate generalization by using recursive strategy. Figure 1 shows the answered of first subject to point $a, b, c$, and $d$.

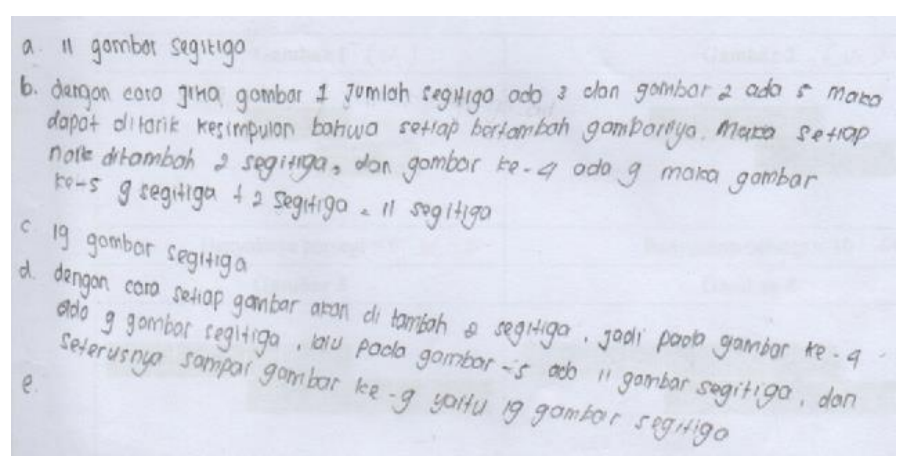

Figure 1: The Answer of Subject 1 to Point $a, b, c$, and $d$

She identified the growth of triangle between step 1 and 2. The number of triangle at step 1 is 3 and step 2 is 5 . She concluded that each step, the number of triangles increase 2 triangles. She answered 11 triangles at step 5. She got the answer from the number of triangle at step 4 and then she added 2 triangles. So, the answer is 19 .

From the figure 1, the first subject also used recursive strategy to reach near generalization. The near 
generalization to point $\mathrm{c}$ and $\mathrm{d}$ on problem 1 . She explained that each step, the number of triangle increase 2 triangles. She revealed that the number of triangle at step 4 is 9 triangles, step 5 is 11 triangles and so on until step 9. So, the answer for point $\mathrm{c}$ and $\mathrm{d}$ is 19 triangles.

The first subject used functional strategy to answered the far generalization to point e and $\mathrm{f}$. At point e, she wrote the number of triangle at step 1 is $a$, step 2 is $a+2$, step 3 is $a+4(a+2+2)$ and step $n$ is $a+(2+2+2 \ldots+2)$. She wrote $a$ as a symbol the number of triangle at step 1 but she didn't understand to use the symbol for the next step. For step 2 and 3, she didn't replace $a$ with the number of triangle at step 1 . Overall, she explained the answer of number triangle step 2 and 3, she answered correctly. The answer for step $n$ was correctly but she didn't explain how many to adding 2 . Overall, she answered the question at point e incorrectly. At point $\mathrm{f}$, she answered $u_{n}=u_{1}+(n-1) 3$. At point $\mathrm{f}$, she didn't explain the answered at point e but she answered with the different things. She tried to find the far generalization through the formula $u_{n}=u_{1}+(n-1) b$ but she didn't answer correctly. From her answer at the task, she wrote the difference is 3 while the correct difference is 2 . At point e, she wrote $a$ as a symbol of step $1\left(u_{1}\right)$ but she wrote $u_{1}$ at point $f$. She didn't consistenly use a symbol.

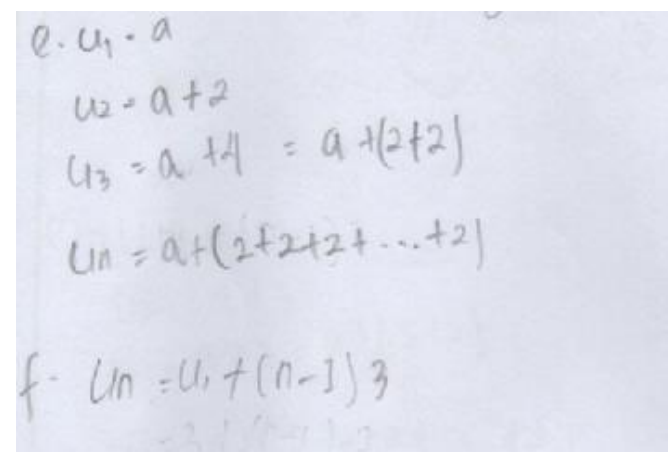

Figure 2: The Answer of Subject 1 to Point $e$ and $f$

According to the results of the task performed by the first subject, she used recursive strategies for immediate generalization. She was able to recognize figural pattern by finding the difference between the given pattern. Then she added the difference into the given pattern to determine the next pattern. She also used recursive strategies for near generalization. She utilized functional strategies for far generalization on each problem but had an incorrectly answer. She identified the growing components of the pattern as well as the constant components but she was not insert the constant component correctly on the rule. Overall, she performed figural reasoning based on the figural pattern task.
B. The reasoning of second subject (low mathematical level)

Problem at generalization task was solved by second subject. The second subject answered the immediate generalization by using recursive strategy. Figure 3 shows the answered of first subject to point $a, b, c$, and $d$.

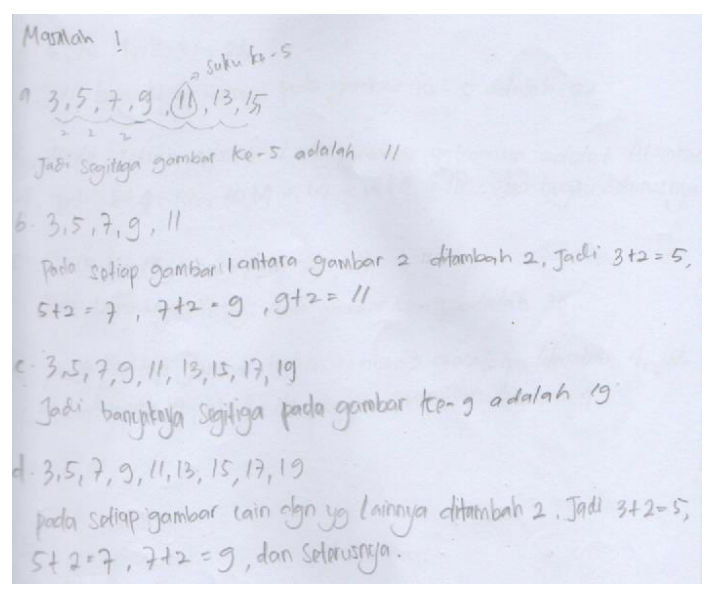

Figure 3: The Answer of Subject 2 in point $a, b, c$ and $d$

She identified the difference of triangle between the given pattern. She transformed the given pattern to number series. She wrote the number of triangle at step 1 until step 7. The difference at number series is 2 . She got it from the number of triangle at step 2 reduced by the number of triangle at step 1 . The number of triangle at step 1 is 3 and step 2 is 5 . She concluded that each step increase 2 triangles. She explain the number of triangle at step 2 from $3+2$, the number of triangle at step 3 from $5+2$, the number of triangle at step 4 from $7+2$, and then the number of triangle at step 5 from 9+2 equal 11 .

From the figure 3 , the second subject also used recursive strategy to reach near generalization. The near generalization to point $c$ and $d$. she wrote the number of triangle at step 1 until step 9 in to number series. She got the number of triangle at step 5 by adding the number of triangle at step 4 with 2 . She answered the question correctly.

The second subject also used functional strategy to answered the far generalization to point $e$ and $f$. At point $e$, she wrote the number of triangle at step 1 is $a$, step 2 is $a+2$, step 3 is $a+4(a+2.2)$ and step $n$ is $a+(2+2+2 \ldots+2)$. She wrote $a$ as a symbol the number of triangle at step 1 but she didn't replace $a$ with the number of triangle at step 1. Overall, she explained the answer of number triangle step 2 and 3, she answered correctly. The answer for step $n$ was incorrectly because she didn't explain how many to adding 2. Overall, she 
answered the question at point e incorrectly. At point $\mathrm{f}$, she answered $u_{n}=u_{1}+(n-1) 3$. At point $f$, she didn't explain the answered at point $e$ but she answered with the different things. She tried to find the far generalization through the formula $u_{n}=u_{1}+(n-1) b$ but she didn't answer correctly. From her answer at the task, she wrote the difference is 3 while the correct difference is 2 . At point $e$, she wrote $a$ as a symbol of step $1\left(u_{1}\right)$ but she wrote $u_{1}$ at point $f$. She didn't consistenly use a symbol.

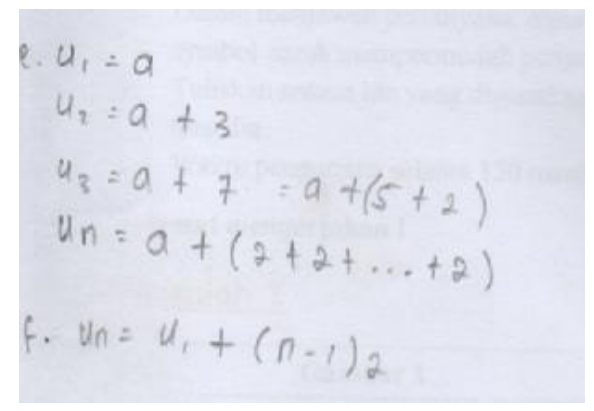

Figure 4: The Answer of Subject 2 in point $a, b, c$ and $d$

The second subject implemented recursive strategies for immediate generalization. Take into account that the first subject recognized figural pattern by finding the difference between the given pattern, the second subject involved numerical pattern. She summed the difference up to the given number from figural pattern to obtain the next pattern. She also employed recursive strategies for near generalization. She implied functional strategies for far generalization but had an incorrect answer. She was able to identify the growing components of the pattern as well as the constant components but she was not insert the constant component correctly on the rule. Overall, she categorized numerical reasoning based on the figural pattern task.

\section{CONCLUSION}

According to the results of the task performed by the first subject, she performed figural reasoning based on the figural pattern task. The second subject categorized numerical reasoning based on the figural pattern task.

\section{REFERENCES}

[1] J. Sidenvall, J. Lithner, and J. Jader, "Students' Reasoning in Mathematics Textbook Task-Solving", International Journal of Mathematical Education in Science and Technology, vol. 46, issue 4, 2015.

[2] D. Sukirwan and T. Herman, "Analysis of Students' Mathematical Reasoning”, Journal of Physics: Conference Series, vol. 948, no. 1, 2014.

[3] E. Rubinstein and R. McGraw, Developing Mathematical Reasoning Among Middle School Immigrant Students: Building on First and Second Language Competencies, 2008 , (http://math.arizona.edu/ cemela/english/content/workingpapers/Rubins tein-Avila\&McGraw-AERA08[1].pdf).

[4] E. Warren and T. Cooper, "Generalising the Pattern Rule for Visual Growth Patterns: Actions that support 8 year olds' thinking”, Educ. Stud. Math, vol. 67, issue 2, pp. 171-185, 2007.

[5] A. Barbosa and I. Vale, "Visualization in pattern generalization: Potential and Challenges", Journal of the European Teacher Education Network, vol. 10, pp. 57-70, 2015.

[6] R. E. Mouhayar and M. Jurdak, "Variation of Student Numerical and Figural Reasoning Approaches by Pattern Generalization Type, Strategy Use and Grade Level", International Journal of Mathematical Education in Science and Technology, vo. 47, issue 2, 2016.

[7] F. D. Rivera and J. R. Becker, "Middle School Children's Cognitive Perceptions of Constructive and Deconstructive Generalizations Involving Linear Figural Patterns", ZDM Mathematics Education, vol. 40, pp. 65-82, 2008.

[8] A. Barbosa, Patterning Problems: Sixth Graders' Ability to Generalize. In M. Pytlak, T. Rowland \& E. Swoboda, Proceedings of the Seventh Congress of the European Society for Research in Mathematics Education, 2011, pp. 420-428. 